\title{
Association of Target Lesion Characteristics Evaluated by Coronary Computed Tomography Angiography and Plaque Debris Distal Embolization During Percutaneous Coronary Intervention
}

\author{
Mayu Nishio, MD, PhD; Yasunori Ueda, MD, PhD; Koshi Matsuo, MD; \\ Masahiko Tsujimoto, MD, PhD; Hiroyuki Hao, MD, PhD; Mitsutoshi Asai, MD, PhD; \\ Takayoshi Nemoto, MD; Mitsuru Wada, MD; Akio Hirata, MD, PhD; Ayaka Murakami, BSc; \\ Kazunori Kashiwase, MD, PhD; Kazuhisa Kodama, MD, PhD
}

\begin{abstract}
Background: The slow-flow or no re-flow phenomenon has been associated with distal embolization, especially of plaque debris, and with unfavorable clinical outcomes. Therefore, we examined the association between the coronary computed tomography angiography (CCTA) findings of the target lesion and distal embolization during percutaneous coronary intervention $(\mathrm{PCl})$.
\end{abstract}

\begin{abstract}
Methods and Results: Consecutive patients ( $n=55$ : 18 unstable angina, 19 stable effort angina, 18 silent ischemia) who underwent $\mathrm{PCl}$ with a filter-type distal protection device after evaluation of the target lesion by CCTA were analyzed. CCTA examined low-attenuation plaque (LAP), positive remodeling (PR), and ring-like enhancement of the target lesion. Distal embolization of thrombus and plaque debris was evaluated by pathological examination of material collected in the filter. Any distal embolization and distal embolization of plaque debris were respectively detected in $75 \%$ and $0 \%$ of patients with LAP or PR alone, in $95 \%$ and $17 \%$ of patients with both LAP and PR, and in $100 \%$ and $27 \%$ of patients with all of LAP, PR and ring-like enhancement. The sensitivity and specificity to predict plaque debris embolization by having both findings of LAP and PR was $100 \%$ and $46 \%$, respectively.
\end{abstract}

Conclusions: The CCTA findings of the target lesion were associated with distal embolization and were very sensitive for predicting plaque debris embolization. (Circ J 2014; 78: 2203-2208)

Key Words: Coronary computed tomography angiography; Distal embolization; Plaque debris; Thrombus

$\mathbf{I}$ t has been reported that the no-/slow-flow phenomenon occurs in $0.5-1 \%$ of cases of elective percutaneous coronary intervention (PCI), ${ }^{1}$ which is extremely deleterious to the result of PCI. Although distal embolization is the supposed cause, ${ }^{2-4}$ the usefulness of distal protection devices has not been established. ${ }^{5,6}$ A major problem with clinical trials to show the usefulness of such devices is selection of high-risk patients. We have previously reported the beneficial effect of a device in a selected high-risk group of acute MI patients with ruptured plaque at the target lesion. ${ }^{7}$ Distal embolization of plaque debris was frequently demonstrated in those patients.

Coronary computed tomography angiography (CCTA) can evaluate the characteristics of coronary plaques. ${ }^{8,9}$ Low-attenuation plaque (LAP), positive remodeling (PR) and ring-like enhancement have been reported as the CCTA findings associ- ated with future coronary events. ${ }^{10,11} \mathrm{We}$ have previously reported that these CCTA findings are associated with the presence of yellow plaque or ruptured plaque on angioscopy. ${ }^{12}$

In the present study, we examined the association between CCTA findings and distal embolization when using a filtertype distal protection device.

\section{Methods}

\section{Study Patients}

We analyzed 55 consecutive patients who underwent CCTA within 2 weeks before PCI using distal protection device between March 2010 and August 2012, excluding 3 patients without adequate CCTA images because of heavy calcification. The association between the CCTA findings of the PCI

Received January 27, 2014; revised manuscript received May 28, 2014; accepted May 29, 2014; released online July 4, 2014 Time for primary review: 29 days

Cardiovascular Division (M.N., Y.U., K.M., M.A., T.N., M.W., A.H., A.M., K. Kashiwase, K. Kodama), Department of Diagnostic Pathology (M.T.), Osaka Police Hospital, Osaka; Department of Surgical Pathology, Hyogo College of Medicine, Nishinomiya (H.H.), Japan

Mailing address: Yasunori Ueda, MD, PhD, FACC, FESC, FJCC, Cardiovascular Division, Osaka Police Hospital, 10-31 Kitayama-cho, Tennoji-ku, Osaka 543-0035 Japan. E-mail: ueda@oph.gr.jp

ISSN-1346-9843 doi:10.1253/circj.CJ-14-0103

All rights are reserved to the Japanese Circulation Society. For permissions, please e-mail: cj@j-circ.or.jp 


\begin{tabular}{|c|c|}
\hline $\mathbf{n}$ & 55 \\
\hline Male sex, n (\%) & $47(85)$ \\
\hline Age, years & $61.4 \pm 7.0$ \\
\hline \multicolumn{2}{|l|}{ Risk factors, n (\%) } \\
\hline Diabetes mellitus & $19(35)$ \\
\hline Hypertension & $46(84)$ \\
\hline Dyslipidemia & $46(84)$ \\
\hline Current smoking & $13(24)$ \\
\hline Body mass index & $25.3 \pm 3.9$ \\
\hline \multicolumn{2}{|l|}{ Lipid profile, mg/dl } \\
\hline Total cholesterol & $181 \pm 32$ \\
\hline LDL-cholesterol & $102 \pm 25$ \\
\hline HDL-cholesterol & $48 \pm 12$ \\
\hline Triglycerides & $159 \pm 112$ \\
\hline Hemoglobin A1c, \% & $6.0 \pm 1.4$ \\
\hline \multicolumn{2}{|l|}{ Diagnosis, n (\%) } \\
\hline Unstable angina & $18(33)$ \\
\hline Stable effort angina & $19(35)$ \\
\hline Silent myocardial ischemia & $18(33)$ \\
\hline \multicolumn{2}{|l|}{ Medications, n (\%) } \\
\hline Statin & $32(58)$ \\
\hline Aspirin & $49(89)$ \\
\hline Clopidogrel/ticlopidine & $40(73)$ \\
\hline ARB/ACEI & $24(44)$ \\
\hline$\beta$-blocker & $15(27)$ \\
\hline Ca-blocker & $24(44)$ \\
\hline
\end{tabular}

Continuous data are presented as mean \pm SD.

ACEI, angiotensin-converting enzyme inhibitor; ARB, angiotensin-receptor blocker; CCTA, coronary computed tomography angiography; HDL, high-density lipoprotein; LDL, low-density lipoprotein; $\mathrm{PCl}$, percutaneous coronary intervention.

target lesion and pathologically examined distal embolization was analyzed. Written informed consent was given by all the patients and the protocol was approved by the Osaka Police Hospital Ethics Committee.

\section{CCTA Examination and Evaluation}

CCTA was performed with a 64-detector row CT scanner (Light speed VCT, GE Healthcare Japan, Tokyo, Japan). All patients with a heart rate $>60$ beats/min were given metoprolol $20 \mathrm{mg}$ orally $2 \mathrm{~h}$ before CT scan. At the time of examination, patients with heart rates $>70$ beats/min were given $2 \mathrm{mg}$ of intravenous propranolol. All patients received $0.3 \mathrm{mg}$ nitroglycerin sublingually immediately before scanning. CCTA data were acquired using X-ray beam collimation (width $0.625 \times 64 \mathrm{~mm}$, gantry rotation time $0.35 \mathrm{~s}$, tube voltage $100-120 \mathrm{mV}$, effective tube current $280-750 \mathrm{~mA}$ using ECG modulation) and the pitch ranged from 0.18 to 0.26 depending on the patient's heart rate. The estimated mean radiation dose was $15-18 \mathrm{mSv}$.

A non-contrast scan was initially performed to determine the anatomic landmarks for the contrast-enhanced study. Immediately thereafter, test bolus tracking with $15 \mathrm{ml}$ of non-ionic contrast agent was performed to calculate the exact arrival time of contrast agent in the coronary arteries, with the region of interest in the proximal part of the ascending aorta. Finally, a contrast-enhanced scan with retrospective ECG gating was performed after administration of contrast medium $(0.8 \mathrm{ml} \cdot \mathrm{kg}$ body weight $\left.{ }^{-1} \cdot 12 \mathrm{~s}^{-1}\right)$ during a single breath hold. Image reconstruc-

\begin{tabular}{|c|c|}
\hline No. of lesions & 55 \\
\hline \multicolumn{2}{|l|}{ Target vessel, n (\%) } \\
\hline Left anterior descending coronary artery & $22(40)$ \\
\hline Left circumflex coronary artery & $9(16)$ \\
\hline Right coronary artery & $24(44)$ \\
\hline \multicolumn{2}{|l|}{ Angiographic findings } \\
\hline AHA/ACC classification, $\mathrm{A} / \mathrm{B} 1 / \mathrm{B} 2 / \mathrm{C}$ & $17 / 20 / 15 / 3$ \\
\hline Diameter stenosis, \% & $67.8 \pm 15.6$ \\
\hline Reference diameter, $\mathrm{mm}$ & $3.2 \pm 0.7$ \\
\hline Lesion length, mm & $22.5 \pm 10.2$ \\
\hline \multicolumn{2}{|l|}{ IVUS findings } \\
\hline Minimum lumen area, $\mathrm{mm}^{2}$ & $2.8 \pm 0.5$ \\
\hline Maximum plaque area, $\mathrm{mm}^{2}$ & $14.1 \pm 4.3$ \\
\hline \multicolumn{2}{|l|}{ CT findings } \\
\hline Low-attenuation plaque, $\mathrm{n}(\%)$ & $34(62)$ \\
\hline Positive remodeling, $n(\%)$ & $35(64)$ \\
\hline Ring-like enhancement, $\mathrm{n}(\%)$ & $20(36)$ \\
\hline \multicolumn{2}{|l|}{ Procedural data } \\
\hline No. of aspiration thrombectomies & $0.3 \pm 0.8$ \\
\hline Stent diameter, $\mathrm{mm}$ & $3.2 \pm 0.4$ \\
\hline Total stent length, mm & $27.6 \pm 16.4$ \\
\hline Maximum inflation pressure, atm & $17.2 \pm 3.5$ \\
\hline
\end{tabular}

Continuous data are presented as mean \pm SD.

IVUS, intravascular ultrasound. Other abbreviations as in Table 1.

tion was performed with image-analysis software (CardIQ, GE Healthcare Japan) on a dedicated computer workstation (Advantage Workstation Ver. 4.2, GE Healthcare Japan). A standard kernel was used as the reconstruction filter. Depending on heart rate, either a half-scan or multi-segment reconstruction algorithm was selected or the optimal cardiac phase with the least motion artifacts was chosen individually.

Coronary arterial remodeling was evaluated by cross-sectional images. The remodeling index was calculated as the ratio of outer vessel diameter at the plaque site to the mean diameter of proximal and distal reference sites. PR was defined as remodeling index $>1.05 .^{13}$ The $\mathrm{CT}$ attenuation value of a plaque was measured at 5 points and averaged. LAP was defined as a CT attenuation value $<40$ HU. ${ }^{14}$ Ring-like enhancement was defined as previously reported. ${ }^{15}$ Briefly, it is the presence of a ring with high attenuation around certain plaque with an attenuation value higher than that of adjacent plaque but no greater than $130 \mathrm{HU}$ to exclude calcium deposition.

\section{Catheterization and PCI}

Catheterization was performed by femoral, brachial, or radial artery approach using 6 or 7Fr sheath and catheters. Coronary angiogram was recorded by the Innova Cardiovascular imaging system (GE Healthcare Japan). Intravascular ultrasound (IVUS) is routinely used as a guide for all PCI procedures in our hospital. Distal protection was performed in all enrolled patients with a filter-type distal protection device Filtrap (Lifeline, Tokyo, Japan) during the PCI procedure. Filtrap is a singleuse steerable 0.014" fixed-wire equipped with a filter basket. During the PCI procedure, plaque debris and thrombus are collected by the filter basket, while blood flows freely through the porous membrane of the filter. At the end of procedure, the filter retaining the embolic material is removed from the target vessel and processed for pathologic examination. Device suc- 

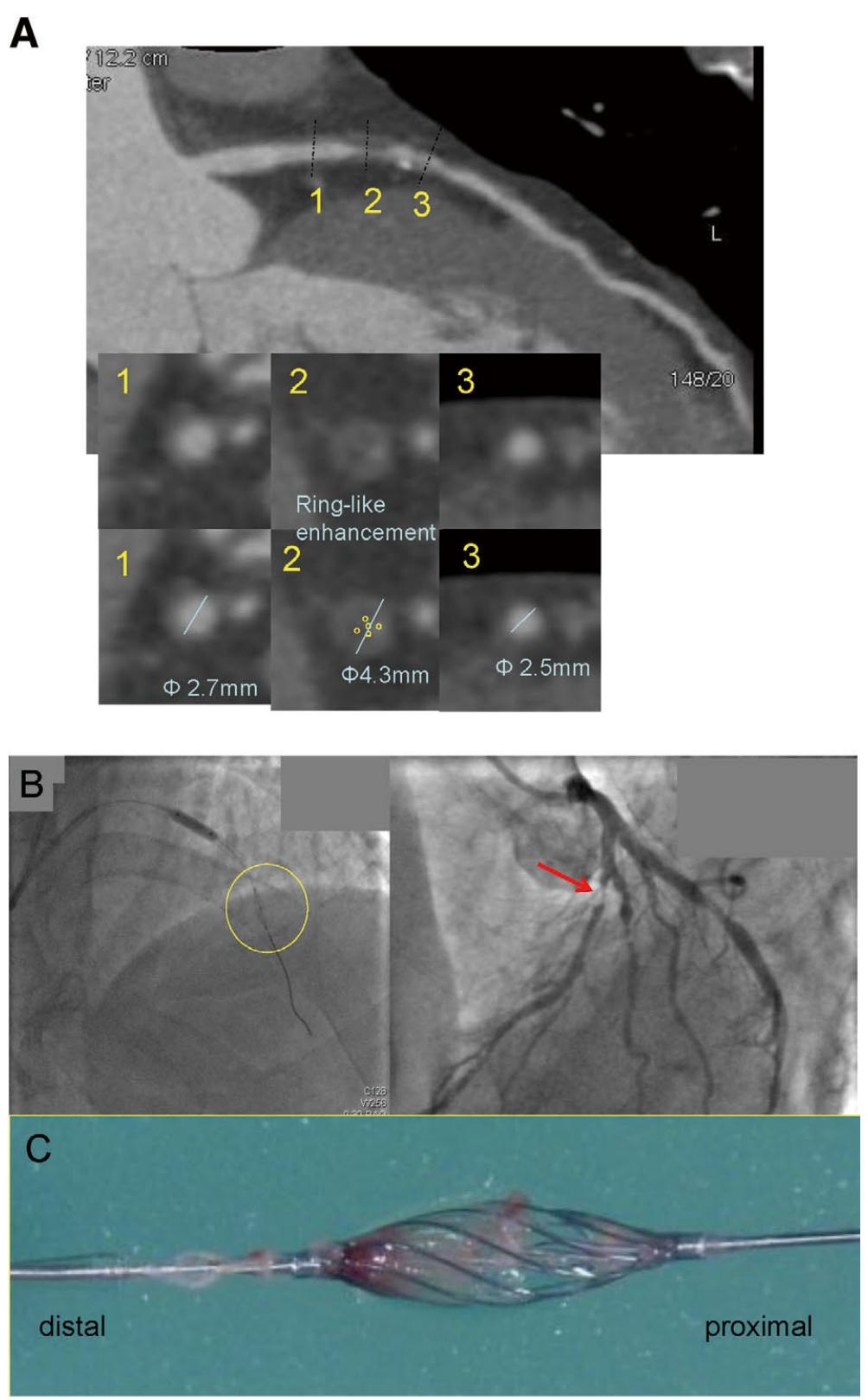

D

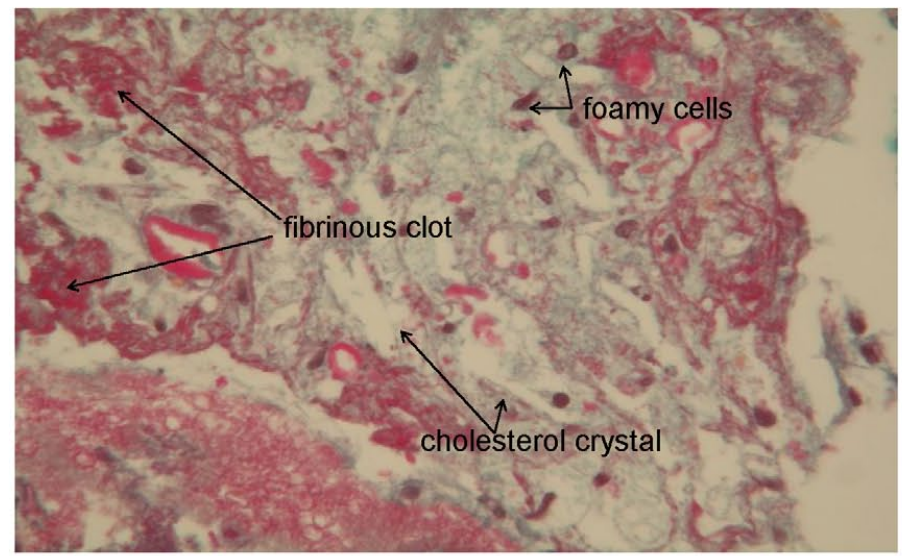

$\mathrm{x} 100$
Figure 1. Representative case of a 68-yearold male patient with unstable angina. Coronary computed tomography angiography (CCTA) detected a significant stenosis in the proximal left anterior descending coronary artery with a plaque that had low attenuation (CT value=24 HU; the sites of CT value measurement are shown by yellow circles), positive remodeling, and ring-like enhancement (A). Percutaneous coronary intervention ( $\mathrm{PCl}$ ) of the stenotic lesion ( $\mathbf{B}$, red arrow) was performed with a filter-type distal protection device (B, yellow circle). Plaque debris was macroscopically detected in the filter of the distal protection device after $\mathrm{PCI}$ (C). Pathologically, the debris contains platelet and fibrinous clot, foamy cells, and cholesterol crystals (D). 




Figure 2. Frequency of distal embolization at the site of various coronary computed tomography angiography (CCTA) findings. Distal embolization of plaque debris was detected only when the target lesion had both findings of low-attenuation plaque (LAP) and positive remodeling (PR). The sensitivity and specificity to predict plaque debris embolization by having both findings was $100 \%$ and $46 \%$, respectively. The sensitivity and specificity to predict plaque debris embolization by having all 3 findings of LAP, PR, and ring-like enhancement was $57 \%$ and $77 \%$, respectively.

cess was defined as successful deployment and retrieval of Filtrap without angiographic complications such as dissection and vasospasm. The no-/slow-flow phenomenon was defined as the apparent reduction of coronary flow speed observed on angiography (ie, TIMI flow grade $<3$ ) at the end of the PCI procedure after removal of the distal protection device. Filter no-/slow-flow was defined as the no-/slow-flow phenomenon before removal of the distal protection device.

\section{Pathologic Examination and Evaluation}

The tip of the Filtrap device (filter) was cut and processed for pathologic examination. The collected material was gently removed from the filter and processed for histological examination. It was stained with hematoxylin-eosin, Masson trichrome, and elastica van Gieson. Two expert pathologists blinded to the patients' characteristics analyzed the specimens independently, and in case of disagreement, they discussed until they reached consensus. The specimens were analyzed for the presence of thrombus and plaque debris. Thrombus was detected by the presence of fibrin or platelets. Plaque debris was detected by the presence of atherosclerotic plaque tissue (extracellular matrix, necrotic gruel, fibrous fragments, foam cells, cholesterin crystals, or calcium deposits). Distal embolization in the present study was defined as the presence of captured material (thrombus or plaque debris) in the filter device.

\section{Statistical Analysis}

Continuous data are presented as mean \pm SD. Comparisons between groups were done by unpaired Student's t-test, the chisquare test, or Kruskal-Wallis test. $\mathrm{P}<0.05$ was regarded as statistically significant. Analysis was done with SPSS version $16.0 \mathrm{~J}$ for Windows (SPSS Inc, Chicago, IL, USA).

\section{Results}

\section{Study Patients}

Positioning of the Filtrap was attempted in 55 patients and device success was attained in all of them. Although filter no-/ slow-flow was detected in $11(20 \%)$ patients, the no-/slowflow phenomenon after filter removal was not detected in any patient. Patients' characteristics are presented in Table 1 and lesion and procedural characteristics are presented in Table 2. A representative case is presented in Figure 1.

\section{CCTA and Pathologic Findings}

Distal embolization was detected in 42 (76\%) patients. Thrombus embolization was detected in $35(64 \%)$ patients and plaque debris embolization was detected in $7(13 \%)$ patients. The incidence of distal embolization (thrombus or plaque debris) in the patients with each combination of CCTA findings (LAP, $\mathrm{PR}$, and/or ring-like enhancement) is presented in Figure 2. Distal embolization of plaque debris was detected only when the target lesion had both findings of LAP and PR. The sensitivity and specificity to predict plaque debris embolization by having both findings of LAP and PR was $100 \%$ and 46\%, respectively. The sensitivity and specificity to predict plaque debris embolization by having all 3 findings of LAP, PR, and ring-like enhancement was $57 \%$ and $77 \%$, respectively.

The minimum lumen area $(2.8 \pm 0.5,2.9 \pm 0.6,2.8 \pm 0.5$, $\left.2.7 \pm 0.5 \mathrm{~mm}^{2}\right)$ and maximum plaque area $(14.1 \pm 4.3,9.6 \pm 1.7$, $13.9 \pm 5.4,14.3 \pm 4.4 \mathrm{~mm}^{2}$ ) measured by IVUS were not different among the patients with each combination of CCTA findings (none, LAP or PR, both LAP and PR, and all of LAP, PR, and ring-like enhancement).

Although the serum troponin T level on next day after PCI was not different among the patients with each combination of CCTA findings, the incidence of filter no-/slow-flow was higher in the patients with more CCTA findings (Table 3). Among non-ACS patients, filter no-/slow-flow was more frequently detected in the patients with plaque debris embolization than in those without ( $83 \%$ vs. $10 \%, \mathrm{P}=0.004)$.

\section{Discussion}

According to the results of the present study, distal embolization of plaque debris was detected in $17 \%$ of patients who had both of LAP and PR and in $27 \%$ of patients who had all of LAP, PR and ring-like enhancement signs on CCTA. Remarkably, distal embolization of plaque debris was detected only when

\begin{tabular}{|c|c|c|c|c|}
\hline & None & LAP/PR & LAP+PR & LAP+PR+Ring \\
\hline No. of lesions & 14 & 8 & 18 & 15 \\
\hline Incidence of filter no-/slow-flow, n (\%) & $0(0)$ & $1(13)$ & $5(28)$ & $5(33)^{*}$ \\
\hline Serum troponin $\mathrm{T}$ level, ${ }^{\dagger} \mathrm{ng} / \mathrm{ml}$ & $0.08 \pm 0.09$ & $0.29 \pm 0.64$ & $0.06 \pm 0.11$ & $0.15 \pm 0.23$ \\
\hline
\end{tabular}

${ }^{*} \mathrm{P}=0.02$ vs. none. Continuous data are presented as mean $\pm \mathrm{SD} .^{\dagger}$ Measured on the day after $\mathrm{PCl}$.

CCTA, coronary computed tomography angiography; LAP, low-attenuation plaque; PR, positive remodeling; Ring, ring-like enhancement. 
the target lesion had both findings of LAP and PR.

\section{Factors Associated With Embolization}

According to previous reports, distal micro-embolization occurs in $0-70 \%,{ }^{16}$ and the no-/slow-flow phenomenon in $0.5-$ $1 \%$ of PCI cases. ${ }^{17}$ As we have previously reported, ${ }^{18}$ the no-/ slow-flow phenomenon occurs more frequently in patients with ruptured plaque at the target lesion of PCI than in patients without it; and distal embolization of plaque debris is detected more frequently in those patients. Therefore, embolization of plaque debris may be an important cause of the no-/slow-flow phenomenon, which has been associated with deterioration of left ventricular function and worsening of clinical outcome. In the present study, the presence of LAP, PR and ring-like enhancement was demonstrated as predictive of plaque debris embolization. These CCTA findings were significantly associated with the presence of ruptured plaque, as we previously reported. ${ }^{12}$ In both that report and the present study, the incidence of ruptured plaque and that of plaque debris embolization was $25 \%$ and $17 \%$, respectively, in patients who had both LAP and PR, and in $60 \%$ and $27 \%$, respectively, of patients with all 3 findings of LAP, PR and ring-like enhancement. This is consistent with our previous finding that plaque debris embolization and the no-/slow-flow phenomenon occurs more frequently in patients with ruptured plaque., ${ }^{7,18}$

There are some previous reports on the frequency of thrombus or plaque debris embolization. Embolization of thrombus was pathologically detected with a distal protection device in $95 \%$ of acute MI patients, ${ }^{7}$ in $79 \%$ of unstable angina patients, ${ }^{19}$ and in $70 \%$ of stable angina patients. ${ }^{19}$ The embolization of plaque debris was pathologically detected with a distal protection device in $69 \%$ of acute MI patients, ${ }^{7}$ in $73 \%$ of ST-elevation MI patients, ${ }^{20}$ in $21 \%$ of unstable angina patients, ${ }^{19}$ and in $30 \%$ of stable angina patients. ${ }^{19}$ The embolization of thrombus or plaque debris was detected in $100 \%$ of acute MI patients by high-intensity transient signals (HITS) using Doppler guide wire. ${ }^{1}$ These previously reported results are consistent with the results of the present study.

The clinical outcome of distal embolization could not be evaluated in the present study, because the distal protection device should have prevented real embolization into microvessels. Indeed, peri-procedural MI, as shown by the serum troponin T levels on the day after PCI not being different among the patients with each combination of CCTA findings. However, the incidence of filter no-/slow-flow was significantly associated with the CCTA findings. Furthermore, our findings were consistent with a recent report in which LAP and PR were detected more often in the patients who suffered no-/slowflow than in those who did not. ${ }^{21}$ Therefore, these very simple markers (LAP and PR) could be important for evaluating the risk of the no-/slow-flow phenomenon during PCI, which can be performed less invasively by magnetic resonance imaging, ${ }^{22}$ and might be prevented by a distal protection device or more simply by laser angioplasty. ${ }^{23}$ These issues are expected to be investigated in the near future.

\section{Study Limitations}

Our study population was limited to those who underwent both CCTA and PCI and thus a selection bias might be possible. Some of the thrombus detected in the filter device might have been from-situ thrombus formation rather than embolized thrombus, which might have resulted in overestimation of the frequency of thrombus embolization; therefore, we focused more on plaque debris embolization. We did not measure plaque volume, which have influenced the results, because the accu- racy of plaque volume measurement by CCTA is still uncertain. Because this study was not designed to evaluate IVUS findings, adequate IVUS images were not available for study patients examined by various IVUS systems from different manufacturers, but mainly by manual pullback. We have not demonstrated the effectiveness of distal protection devices to prevent the no-/slow-flow phenomenon, which must be demonstrated in future randomized controlled clinical trials.

\section{Conclusions}

CCTA findings were well associated with distal embolization. The presence of both findings of LAP and PR was highly sensitive for predicting plaque debris embolization.

\section{Disclosures}

None of the authors has any conflict to disclose.

\section{Funding}

None.

\section{References}

1. Good CW, Blankenship JC, Scott TD, Skelding KA, Berger PB, Wood GC. Feasibility and safety of ad hoc percutaneous coronary intervention in the modern era. J Invasive Cardiol 2009; 21: 194200.

2. Virmani R, Burke AP, Farb A, Kolodgie FD. Pathology of the vulnerable plaque. J Am Coll Cardiol 2006; 47(Suppl): C13-C18.

3. Kawamoto T, Okura H, Koyama Y, Toda I, Taguchi H, Tamita K, et al. The relationship between coronary plaque characteristics and small embolic particles during coronary stent implantation. J Am Coll Cardiol 2007; 50: 1635-1640.

4. Bose D, von Birgelen C, Zhou XY, Schermund A, Philipp S, Sack S, et al. Impact of atherosclerotic plaque composition on coronary microembolization during percutaneous coronary interventions. Basic Res Cardiol 2008; 103: 587-597.

5. Gick M, Jander N, Bestehorn HP, Kienzle RP, Ferenc M, Werner K, et al. Randomized evaluation of the effects of filter-based distal protection on myocardial perfusion and infarct size after primary percutaneous catheter intervention in myocardial infarction with and without st-segment elevation. Circulation 2005; 112: 1462-1469.

6. Stone GW, Webb J, Cox DA, Brodie BR, Qureshi M, Kalynych A, et al. Distal microcirculatory protection during percutaneous coronary intervention in acute ST-segment elevation myocardial infarction: A randomized controlled trial. JAMA 2005; 293: 1063-1072.

7. Mizote I, Ueda Y, Ohtani T, Shimizu M, Takeda Y, Oka T, et al. Distal protection improved reperfusion and reduced left ventricular dysfunction in patients with acute myocardial infarction who had angioscopically defined ruptured plaque. Circulation 2005; 112: 1001 1007.

8. Schmid M, Pflederer T, Jang IK, Ropers D, Sei K, Daniel WG, et al. Relationship between degree of remodeling and CT attenuation of plaque in coronary atherosclerotic lesions: An in-vivo analysis by multi-detector computed tomography. Atherosclerosis 2008; 197: 457-464.

9. Pohle K, Achenbach S, Macneill B, Ropers D, Ferencik M, Moselewski F, et al. Characterization of non-calcified coronary atherosclerotic plaque by multi-detector row CT: Comparison to IVUS. Atherosclerosis 2007; 190: 174-180.

10. Inoue F, Sato Y, Matsumoto N, Tani S, Uchiyama T. Evaluation of plaque texture by means of multislice computed tomography in patients with acute coronary syndrome and stable angina. Circ J 2004; 68: $840-844$.

11. Motoyama S, Kondo T, Sarai M, Sugiura A, Harigaya H, Sato T, et al. Multislice computed tomographic characteristics of coronary lesions in acute coronary syndromes. J Am Coll Cardiol 2007; 50: 319-326.

12. Nishio M, Ueda Y, Matsuo K, Asai M, Nemoto T, Hirata A, et al. Detection of disrupted plaques by coronary CT: Comparison with angioscopy. Heart 2011; 97: $1397-1402$.

13. Achenbach S, Ropers D, Hoffmann U, MacNeill B, Baum U, Pohle $\mathrm{K}$, et al. Assessment of coronary remodeling in stenotic and nonstenotic coronary atherosclerotic lesions by multidetector spiral computed tomography. J Am Coll Cardiol 2004; 43: 842-847. 
14. Kitagawa T, Yamamoto H, Horiguchi J, Ohhashi N, Tadehara F, Shokawa T, et al. Characterization of noncalcified coronary plaques and identification of culprit lesions in patients with acute coronary syndrome by 64 -slice computed tomography. JACC Cardiovasc Imaging 2009; 2: 153-160.

15. Tanaka A, Shimada K, Yoshida K, Jissyo S, Tanaka H, Sakamoto M, et al. Non-invasive assessment of plaque rupture by 64 -slice multidetector computed tomography: Comparison with intravascular ultrasound. Circ J 2008; 72: 1276-1281.

16. Hermann J. Peri-procedural myocardial injury: 2005 update. Eur Heart J 2005; 26: 2493-2519.

17. Mattichak SJ, Dixon SR, Shannon F, Boura JA, Safian RD. Failed percutaneous coronary intervention: A decade of experience in 21,000 patients. Catheter Cardiovasc Interv 2008; 71: 131-137.

18. Matsuo K, Ueda Y, Tsujimoto M, Hao H, Nishio M, Hirata A, et al. Ruptured plaque and large plaque burden are risks of distal embolisation during percutaneous coronary intervention: Evaluation by angioscopy and virtual histology intravascular ultrasound imaging. EuroIntervention 2013; 9: 235-242.

19. Angelini A, Rubartelli P, Mistrorigo F, Della Barbera M, Abbadessa
F, Vischi M, et al. Distal protection with a filter device during coronary stenting in patients with stable and unstable angina. Circulation 2004; 110: 515-521.

20. Dewey M, Zimmermann E, Deissenrieder F, Laule M, Dübel HP, Schlattmann P, et al. Noninvasive coronary angiography by 320-row computed tomography with lower radiation exposure and maintained diagnostic accuracy: Comparison of results with cardiac catheterization in a head-to-head pilot investigation. Circulation 2009; 120: 867875.

21. Kodama T, Kondo T, Oida A, Fujimoto S, Narula J. Computed tomographic angiography-verified plaque characteristics and slow-flow phenomenon during percutaneous coronary intervention. JACC Cardiovasc Inter 2012; 5: 636-643.

22. Noguchi T, Yamada N, Kawasaki T, Tanaka A, Yasuda S. Detection of high-risk atherosclerotic plaques by magnetic resonance imaging. Circ J 2013; 77: 1975-1983.

23. Shishikura D, Otsuji S, Takiuchi S, Fukumoto A, Asano K, Ikushima $\mathrm{M}$, et al. Vaporizing thrombus with excimer laser before coronary stenting improves myocardial reperfusion in acute coronary syndrome. Circ J 2013; 77: 1445-1452. 